\title{
Geodiversidade no ambiente escolar: uma análise de teses e dissertações nacionais
}

\author{
Geodiversity in the school environment: an analysis of national theses and dissertations \\ Geodiversidad en el entorno escolar: un análisis de tesis y disertaciones nacionales
}

Recebido: 06/11/2021 | Revisado: 12/11/2021 | Aceito: 17/11/2021 | Publicado: 28/11/2021

\author{
Jhenys Maiker Santos \\ ORCID: https://orcid.org/0000-0002-2409-1547 \\ Universidade Federal do Ceará, Brasil \\ E-mail: jhenysmaiker@gmail.com \\ Patrícia da Cunha Gonzaga Silva \\ ORCID: https://orcid.org/0000-0002-9602-495X \\ Universidade Federal do Piauí, Brasil \\ E-mail: patriciagonzaga18@hotmail.com \\ Paulo Victor de Oliveira \\ ORCID: https://orcid.org/0000-0002-1841-9999 \\ Universidade Federal do Piauí, Brasil \\ E-mail: victoroliveira@ufpi.edu.br
}

\begin{abstract}
Resumo
Este trabalho tem como objetivo investigar como o ensino de Geodiversidade está presente em dissertações e teses dos programas de pós-graduação do Brasil (2009-2019). Foi realizada uma pesquisa quali-quantitativa, de cunho bibliográfico, cujos dados foram coletados no mês de novembro de 2020, na Biblioteca Digital Brasileira de Teses e Dissertações, através de configurações específicas de pesquisa. Foram encontrados 17 trabalhos, dos quais 16 destes são dissertações e apenas um trata-se de tese, apresentados em programas de pós-graduações, defendidas entre 2009 a 2019, em 15 instituições superiores, a saber: UFSC, USP, PUC-Rio, UFRGS, UEPG, UNESP, UFSCar, UFV, UEL, UFSM, UFC, UFRN, UFPE, UFTM e UNIOESTE. De todos os trabalhos, evidenciamos que, em somente um deles, a temática da Geodiversidade é abordada no ambiente escolar. Deste modo, pode-se concluir que a temática da Geodiversidade está presente em alguns trabalhos, mas de maneira ainda tímida e incipiente, especialmente quando se trata do ensino da Geodiversidade.
\end{abstract}

Palavras-chave: Ensino; Educação básica; Geodiversidade; Pós-graduação.

\begin{abstract}
This work aims to investigate how the teaching of Geodiversity is present in dissertations and theses of graduate programs in Brazil (2009-2019). A quali-quantitative research of bibliographic nature was carried out, whose data were collected in the month of November 2020, at the Biblioteca Digital Brasileira de Teses e Dissertações, through specific research configurations. 17 papers were found, of which 16 are dissertations and only one is a thesis presented in graduate programs, defended between 2009 and 2019, in 15 higher education institutions, namely: UFSC, USP, PUC-Rio, UFRGS, UEPG, UNESP, UFSCar, UFV, UEL, UFSM, UFC, UFRN, UFPE, UFTM e UNIOESTE. Of all the works, only one really addresses the theme of geovidersity in the school environment. Thus, it can be concluded that the theme of Geodiversity is present in some works, but in a timid and incipient way, especially when it comes to the teaching of Geodiversity.
\end{abstract}

Keywords: Teaching; Basic education; Geodiversity; Postgraduate.

\section{Resumen}

Este trabajo tiene como objetivo investigar cómo la enseñanza de la Geodiversidad está presente en disertaciones y tesis de programas de posgrado en Brasil (2009-2019). Se realizó una investigación bibliográfica cualitativocuantitativa, cuyos datos fueron recolectados en noviembre de 2020, en la Biblioteca Digital Brasileña de Tesis y Disertaciones, a través de escenarios de investigación específicos. Se encontraron 17 trabajos, de los cuales 16 son disertaciones y solo uno es tesis, presentado en programas de posgrado, defendidos entre 2009 y 2019 , en 15 instituciones superiores, a saber: UFSC, USP, PUC-Rio, UFRGS, UEPG, UNESP, UFSCar, UFV, UEL, UFSM, UFC, UFRN, UFPE, UFTM y UNIOESTE. De todos los trabajos, mostramos que, solo en uno de ellos, la temática de la Geodiversidad se aborda en el ámbito escolar. Así, se puede concluir que el tema de la Geodiversidad está presente en algunas obras, pero de forma tímida e incipiente, especialmente cuando se trata de la enseñanza de la Geodiversidad. Palabras clave: Docencia; Educación básica; Geodiversidad; Posgrado. 


\section{Introdução}

Os primeiros conceitos de Geodiversidade datam do início dos anos 1990 em contraponto aos de biodiversidade que não levam em consideração os elementos abióticos (Serrano Cañadas; Ruyz Flaño, 2007).

Vários autores vêm tentando conceituar Geodiversidade, limitando-se apenas às diversidades de rochas, fósseis, minerais, solos, sedimentos e acidentes geográficos, bem como os seus processos de formação (Kiernan, 2001; Sharples, 2002; Gray, 2004; Azevedo, 2007).

Brilha (2005) adota a definição proposta pela Royal Society for Nature Conservation do Reino Unido, de que a Geodiversidade consiste na variedade de ambientes geológicos, fenômenos e processos ativos que dão origem às paisagens, rochas, minerais, fósseis, solos e outros depósitos superficiais que são o suporte para a vida na Terra.

Embora haja outras numerosas definições para o conceito, nenhuma vem acompanhada de um método de estudo e com a sua aplicação teórica à realidade de um território ou ambiente, (Carcavilla et al., 2008), pois tal conceito, aplicado em um determinado local, seria inteiramente inviável aplica-lo a outro.

Por sua vez, Souza et al. (2007) elencam uma série de fatores e medidas que contribuem para o reconhecimento da Geodiversidade como, elemento de suma importância para a educação, ciência e cultura. Em concordância com Xavier, Meneses \& Cavalcante (2017) o ensino e a divulgação da Geodiversidade são pouco difundidos no âmbito educacional, bem como na sociedade em geral, diferentemente do que acontece com a biodiversidade.

É importante evidenciar que, documentos normativos do ensino escolar brasileiro, a exemplo dos Parâmetros Curriculares Nacionais (PCN), Parâmetros Curriculares Nacionais para o Ensino Médio (PCNEM), Base Nacional Comum Curricular (BNCC), dentre outros, não há citação da palavra Geodiversidade, apesar das inúmeras menções sobre assuntos relacionados ao meio ambiente.

Alencar et al (2012) relatam que, embora os temas relacionados à dinâmica da Terra (para o ensino fundamental) estejam inseridos em duas disciplinas como Ciências e Geografia, eles permanecem sendo tratados de modo superficial e desconectado com a realidade dos alunos, possivelmente devido à falta de preparo e domínio dos profissionais em transmitir o conhecimento, o que conduz a uma subvaloração de sua importância.

É perceptível que estudos e projetos de proteção e conscientização em relação a esse patrimônio dão maior ênfase aos elementos vivos em detrimentos dos elementos não vivos (Florentino Junior, 2018). Deste modo, o presente trabalho teve como objetivo investigar de que forma a Geodiversidade está sendo aplicada ao ensino básico, tendo como base os trabalhos de dissertações e teses dos programas de pós-graduações do Brasil.

\section{Metodologia}

Inicialmente os dados foram coletados no mês de novembro de 2020, na Biblioteca Digital Brasileira de Teses e Dissertações (BDTD) no endereço eletrônico http://bdtd.ibict.br/vufind/, pelo fato da mesma se tratar de um banco de dados nacional hospedando teses e dissertações do Brasil.

Foi utilizado as seguintes configurações: i) busca avançada; ii) buscar por "Geodiversidade" em todos os campos; iii) adicionou-se mais um campo; iv) buscar por: "educação" em todos os campos; v) correspondência da busca: em "TODOS os termos" e por fim, vi) ilustrado: "sem preferência". Com o intuito de maximizar os resultados, bem como para ter uma visão geral acerca da realidade no que diz respeito à Geodiversidade no ambiente escolar, optou-se por não delimitar os anos de busca.

Foi realizado o download de todos os trabalhos e uma análise geral no que diz respeito ao: i) tipo de trabalho; ii) ano de defesa; iii) título; iv) autor; v) instituição; vi) programa; e vii) área de concentração.

Posteriormente, para a análise dos trabalhos adotou-se uma abordagem qualitativa, de cunho bibliográfico, realizando- 
se a análise de conteúdo, conforme Bardin (1977), adotando a pré-análise, exploração do material, tratamento dos dados obtidos e interpretação, por vezes se valendo de dados quantitativos.

- Na pré-análise, foi realizada uma leitura dos resumos dos trabalhos, na qual os mesmos foram triados em conformidade com as configurações da pesquisa.

- Na exploração do material, os trabalhos foram codificados e categorizados em conformidade com as similaridades das unidades de registros que nesse caso optou-se por serem seus objetivos, dessa forma ficando mais claras as unidades de contextos. Por se tratar de resumos, a enumeração foi realizada através da presença e/ou ausência dos termos "Geodiversidade" e "educação", definidas anteriormente.

- No tratamento dos dados obtidos e interpretação, foram realizadas inferências baseadas nas categorias e agrupamentos dos dados selecionados.

\section{Resultados}

\subsection{Apresentação geral dos dados}

A partir da busca na plataforma BDTD com as devidas configurações, foram encontrados 18 trabalhos. Após a análise e triagem percebeu-se que uma dissertação se repetia por duas vezes, uma delas foi desconsiderada, dessa forma totalizando apenas 17. Foi elaborada uma tabela na qual os trabalhos foram nomeados em códigos (T) de acordo com a ordem apresentada (T1 a T17) para sua melhor interpretação (Tabela 1), qualitativamente os trabalhos foram analisados, enquadrados e agrupados por categorias de acordo com suas afinidades (Tabela 2).

Dos 17 trabalhos, 16 são dissertações e apenas um trata-se de tese. Com exceção de uma instituição privada, as demais são públicas. Os anos de defesa são entre 2009 e 2019, em 15 instituições superiores, a saber: UFSC, USP, PUC-Rio, UFRGS, UEPG, UNESP, UFSCar, UFV, UEL, UFSM, UFC, UFRN, UFPE, UFTM e UNIOESTE.

Em relação aos programas de pós-graduação, foram identificados seis na área de Geografia, quatro de Geociências e os demais apenas um em cada área, a saber: Educação, Sustentabilidade na Gestão Ambiental, Solos e Nutrição de Plantas, Profissionalizante em Patrimônio Cultural, Desenvolvimento e Meio Ambiente, Turismo, Ciência e Tecnologia Ambiental.

Tabela 1. Trabalhos identificados na base da BDTD como resultados da pesquisa.

\begin{tabular}{|c|c|c|c|c|c|c|c|}
\hline & TIPO & ANO & TÍTULO & AUTOR & INSTITUIÇÃO & PROGRAMA & $\begin{array}{c}\text { ÁREA DE } \\
\text { CONCENTRAÇÃO }\end{array}$ \\
\hline T1 & Dissertação & 2012 & $\begin{array}{l}\text { A Paisagem de Itapema: } \\
\text { estudo da } \\
\text { Geodiversidade para a } \\
\text { educação ambiental e o } \\
\text { geoturismo }\end{array}$ & $\begin{array}{l}\text { Cristina } \\
\text { Covello }\end{array}$ & UFSC & $\begin{array}{c}\text { Pós-Graduação em } \\
\text { Geografia }\end{array}$ & $\begin{array}{c}\text { Utilização e } \\
\text { Conservação dos } \\
\text { Recursos Naturais }\end{array}$ \\
\hline $\mathbf{T 2}$ & Dissertação & 2014 & $\begin{array}{c}\text { Avaliação da } \\
\text { Geodiversidade em São } \\
\text { Sebastião - SP, como } \\
\text { Patrimônio Geológico }\end{array}$ & $\begin{array}{l}\text { Fernanda } \\
\text { Coyado } \\
\text { Reverte }\end{array}$ & USP & $\begin{array}{c}\text { Pós-Graduação em } \\
\text { Geologia }\end{array}$ & $\begin{array}{l}\text { Mineralogia } \\
\text { Experimental e } \\
\text { Aplicada }\end{array}$ \\
\hline T3 & Dissertação & 2013 & $\begin{array}{l}\text { Professores no museu da } \\
\text { Geodiversidade: o capital } \\
\text { cultural nas percepções e } \\
\text { expectativas da relação } \\
\text { museu x escola }\end{array}$ & $\begin{array}{l}\text { Eveline } \\
\text { Milani } \\
\text { Romeiro } \\
\text { Pereira } \\
\text { Aracri }\end{array}$ & PUC-Rio & $\begin{array}{c}\text { Pós-Graduação em } \\
\text { Educação }\end{array}$ & $*$ \\
\hline T4 & Dissertação & 2015 & $\begin{array}{l}\text { Geoconservação em } \\
\text { grandes cidades e } \\
\text { proposição dos } \\
\text { itinerários geológicos de }\end{array}$ & $\begin{array}{l}\text { Rodrigo } \\
\text { Cybis } \\
\text { Fontana }\end{array}$ & UFRGS & $\begin{array}{l}\text { Pós-Graduação em } \\
\text { Geociências }\end{array}$ & $*$ \\
\hline
\end{tabular}




\begin{tabular}{|c|c|c|c|c|c|c|c|}
\hline & & & $\begin{array}{c}\text { Porto Alegre: } \\
\text { contribuições } \\
\text { metodológicas para } \\
\text { valoração integrada de } \\
\text { unidades geológicas }\end{array}$ & & & & \\
\hline T5 & Dissertação & 2010 & $\begin{array}{c}\text { Trilhas interpretativas } \\
\text { como instrumentos de } \\
\text { Geoturismo e } \\
\text { Geoconservação: caso da } \\
\text { trilha do Salto São Jorge, } \\
\text { Campos Gerais do } \\
\text { Paraná }\end{array}$ & $\begin{array}{l}\text { Ana } \\
\text { Cláudia } \\
\text { Folmann }\end{array}$ & UEPG & $\begin{array}{c}\text { Pós-Graduação em } \\
\text { Geografia }\end{array}$ & Gestão de Território \\
\hline T6 & Dissertação & 2019 & $\begin{array}{l}\text { Inventário, quantificação } \\
\text { e valorização do } \\
\text { geopatrimônio na Bacia } \\
\text { do Rio Corumbataí (SP): } \\
\text { subsídios ao Projeto } \\
\text { Geoparque Corumbataí }\end{array}$ & $\begin{array}{l}\text { André de } \\
\text { Andrade } \\
\text { Kolya }\end{array}$ & UNESP & $\begin{array}{l}\text { Pós-Graduação em } \\
\text { Geociências e } \\
\text { Meio Ambiente }\end{array}$ & * \\
\hline T7 & Dissertação & 2018 & $\begin{array}{c}\text { Inventário e } \\
\text { quantificação dos } \\
\text { geossítios cársticos do } \\
\text { Parque Estadual } \\
\text { Intervales (SP) }\end{array}$ & $\begin{array}{l}\text { Túlio César } \\
\text { Rocha } \\
\text { Camargo }\end{array}$ & UFSCar & $\begin{array}{c}\text { Pós-Graduação em } \\
\text { Sustentabilidade na } \\
\text { Gestão Ambiental }\end{array}$ & Ciências Ambientais \\
\hline T8 & Dissertação & 2009 & $\begin{array}{l}\text { Solos e alternâncias } \\
\text { educativas: pesquisa- } \\
\text { ação na formação de } \\
\text { educadores }\end{array}$ & $\begin{array}{l}\text { Lilian } \\
\text { Messias } \\
\text { Lobo }\end{array}$ & UFV & $\begin{array}{c}\text { Pós-Graduação em } \\
\text { Solos e Nutrição de } \\
\text { Plantas }\end{array}$ & $*$ \\
\hline T9 & Dissertação & 2017 & $\begin{array}{c}\text { Estratégias de } \\
\text { geoconservação no } \\
\text { Parque Estadual da Serra } \\
\text { do Mar - Núcleos } \\
\text { Picinguaba e } \\
\text { Caraguatatuba (SP) }\end{array}$ & $\begin{array}{c}\text { Eliana } \\
\text { Mazzucato }\end{array}$ & USP & $\begin{array}{c}\text { Pós-Graduação em } \\
\text { Geociências }\end{array}$ & $\begin{array}{c}\text { Mineralogia e } \\
\text { Petrologia }\end{array}$ \\
\hline T10 & Dissertação & 2018 & $\begin{array}{l}\text { Geoturismo no norte } \\
\text { paranaense: uma } \\
\text { proposta de georoteiro } \\
\text { para o município de } \\
\text { Santo Antônio da Platina }\end{array}$ & $\begin{array}{l}\text { Euzemar } \\
\text { Florentino } \\
\text { Junior }\end{array}$ & UEL & $\begin{array}{c}\text { Pós-Graduação em } \\
\text { Geografia }\end{array}$ & $\begin{array}{c}\text { Dinâmica Espaço- } \\
\text { Ambiental }\end{array}$ \\
\hline T11 & Dissertação & 2013 & $\begin{array}{c}\text { O patrimônio geológico } \\
\text { no desenvolvimento } \\
\text { territorial em Tibagi, } \\
\text { Paraná }\end{array}$ & $\begin{array}{l}\text { Nair } \\
\text { Fernanda } \\
\text { Mochiutti }\end{array}$ & UFSC & $\begin{array}{c}\text { Pós-Graduação em } \\
\text { Geografia }\end{array}$ & * \\
\hline T12 & Dissertação & 2013 & $\begin{array}{l}\text { Geoturismo: uma } \\
\text { ferramenta auxiliar na } \\
\text { interpretação e } \\
\text { preservação do } \\
\text { patrimônio } \\
\text { geopaleontológico da } \\
\text { região central do Rio } \\
\text { Grande do Sul }\end{array}$ & $\begin{array}{l}\text { Carmen } \\
\text { Terezinha } \\
\text { Barcellos } \\
\text { Lorenci }\end{array}$ & UFSM & $\begin{array}{c}\text { Pós-Graduação } \\
\text { Profissionalizante } \\
\text { em Patrimônio } \\
\text { Cultural }\end{array}$ & $\begin{array}{l}\text { Arquitetura e } \\
\text { Patrimônio }\end{array}$ \\
\hline T13 & Dissertação & 2019 & $\begin{array}{l}\text { Potencialidades para o } \\
\text { desenvolvimento do } \\
\text { geoturismo no Município } \\
\text { de Itapipoca (CE) }\end{array}$ & $\begin{array}{l}\text { Ricardo } \\
\text { Matos } \\
\text { Machado }\end{array}$ & UFC & $\begin{array}{c}\text { Pós-Graduação em } \\
\text { Desenvolvimento e } \\
\text { Meio Ambiente }\end{array}$ & $\begin{array}{c}\text { Proteção Ambiental e } \\
\text { Gestão dos Recursos } \\
\text { Naturais }\end{array}$ \\
\hline T14 & Dissertação & 2015 & $\begin{array}{l}\text { Práticas turísticas em } \\
\text { geossítios: uma avaliação } \\
\text { ambiental no projeto }\end{array}$ & $\begin{array}{c}\text { Janaina } \\
\text { Luciana de }\end{array}$ & UFRN & $\begin{array}{c}\text { Pós-Graduação em } \\
\text { Turismo }\end{array}$ & $\begin{array}{c}\text { Turismo e } \\
\text { Desenvolvimento }\end{array}$ \\
\hline
\end{tabular}




\begin{tabular}{|c|c|c|c|c|c|c|c|}
\hline & & & geoparque Seridó-RN & Medeiros & & & Regional \\
\hline T15 & Tese & 2016 & $\begin{array}{c}\text { Patrimônio geológico e } \\
\text { estratégias de } \\
\text { geoconservação: } \\
\text { popularização das } \\
\text { geociências e } \\
\text { desenvolvimento } \\
\text { territorial sustentável } \\
\text { para o litoral sul de } \\
\text { Pernambuco (Brasil) }\end{array}$ & $\begin{array}{l}\text { Thaís de } \\
\text { Oliveira } \\
\text { Guimarães }\end{array}$ & UFPE & $\begin{array}{l}\text { Pós-Graduação em } \\
\text { Geociências }\end{array}$ & $\begin{array}{c}\text { Geologia Sedimentar } \\
\text { e Ambiental }\end{array}$ \\
\hline T16 & Dissertação & 2018 & $\begin{array}{l}\text { Compartimentos } \\
\text { topomorfológicos e } \\
\text { morfodinâmica da bacia } \\
\text { do Córrego das Lajes: } \\
\text { Uberaba: Triângulo } \\
\text { Mineiro }\end{array}$ & $\begin{array}{l}\text { Francisco } \\
\text { Ferreira } \\
\text { Martins } \\
\text { Neto }\end{array}$ & UFTM & $\begin{array}{c}\text { Pós-Graduação em } \\
\text { Ciência e } \\
\text { Tecnologia } \\
\text { Ambienta }\end{array}$ & Qualidade Ambiental \\
\hline T17 & Dissertação & 2017 & $\begin{array}{c}\text { Caracterização } \\
\text { paisagística dos Parques } \\
\text { Nacionais do Iguaçu e } \\
\text { Iguazu }\end{array}$ & $\begin{array}{c}\text { Juraci } \\
\text { Colpani }\end{array}$ & UNIOESTE & $\begin{array}{c}\text { Pós-graduação em } \\
\text { Geografia }\end{array}$ & $*$ \\
\hline
\end{tabular}

Fonte: BDTD (organizados pelos autores, 2021). *Não listado no trabalho.

\subsection{Análise textual}

Após a análise textual percebeu-se que a maioria dos trabalhos não tinha relação com "Geodiversidade aplicada ao ensino", pois os mesmos foram selecionados pela plataforma BDTD apenas pelo fato de que em algum lugar no decorrer do trabalho foram citados os descritores pesquisados: "Geodiversidade" e "educação".

Posteriormente, os trabalhos foram reagrupados em categorias conforme as suas afinidades por temática abordada, a saber: geoturismo; reconhecimento e inventário da Geodiversidade; valoração/geoconservação; educação ambiental; capital cultural; topografia; caracterização paisagística e solos (Tabela 2).

Tabela 2. Categorias dos trabalhos analisados.

\begin{tabular}{|c|c|c|c|c|c|c|c|c|c|c|c|c|c|c|c|c|c|}
\hline \multirow{2}{*}{ CATEGORIAS } & \multicolumn{17}{|c|}{ TRABALHOS } \\
\hline & $F$ & $\tilde{F}$ & $\hat{\epsilon}$ & $\stackrel{t}{F}$ & 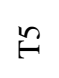 & $\bullet$ & $\hat{F}$ & $\stackrel{\infty}{\oplus}$ & $\hat{\theta}$ & 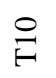 & $\bar{F}$ & $\stackrel{\sim}{F}$ & $\stackrel{m}{F}$ & $\underset{E}{\Xi}$ & $\stackrel{n}{F}$ & $\stackrel{\bullet}{E}$ & $\stackrel{\Xi}{F}$ \\
\hline Geoturismo & $X$ & & & & $\mathrm{X}$ & & $\mathrm{X}$ & & & $\mathrm{X}$ & & $\mathrm{X}$ & $\mathrm{X}$ & $\mathrm{X}$ & $\mathrm{X}$ & & \\
\hline $\begin{array}{l}\text { Reconhecimento e } \\
\text { inventário }\end{array}$ & $\mathrm{X}$ & $\mathrm{X}$ & & & & $\mathrm{X}$ & & & & & & & & & $\mathrm{X}$ & & \\
\hline $\begin{array}{l}\text { Valoração / } \\
\text { geoconservação }\end{array}$ & & & & $\mathrm{X}$ & $\mathrm{X}$ & $\mathrm{X}$ & $\mathrm{X}$ & & $\mathrm{X}$ & $\mathrm{X}$ & $\mathrm{X}$ & & & & $\mathrm{X}$ & & \\
\hline Educação ambiental & $\mathrm{X}$ & & & $\mathrm{X}$ & $\mathrm{X}$ & & $\mathrm{X}$ & & & $\mathrm{X}$ & & & & & $\mathrm{X}$ & & \\
\hline Capital cultural & & & $\mathrm{X}$ & & & & & & & & & & & & & & \\
\hline Topografia & & & & & & & & & & & & & & & & $\mathrm{X}$ & \\
\hline $\begin{array}{l}\text { Caracterização } \\
\text { paisagística }\end{array}$ & & & & & & & & & & & & & & & & & $\mathrm{X}$ \\
\hline Solos & & & & & & & & $\mathrm{X}$ & & & & & & & & & \\
\hline
\end{tabular}

Fonte: Dados da pesquisa (2021). 
Conforme a temática, alguns trabalhos acabavam se enquadrando cumulativamente em outras categorias, isso devido ao foco da pesquisa. A Tabela 2 demostra claramente por meio de suas codificações essas categorias observadas.

\subsubsection{Geoturismo}

Os trabalhos T1, T5, T7, T10, T12, T13, T14 e T15 foram enquadrados na categoria "Geoturismo", pois em algum momento os mesmos citam alguma palavra ou termo com esse sentido.

O trabalho T1, também acumula enquadramento com outras categorias, como: "Reconhecimento e inventário" e “Educação ambiental”. Esta pesquisa trata-se de uma dissertação de mestrado apresentada ao Programa de Pós-Graduação em Geografia, do Departamento de Geociências, Centro de Filosofia e Ciências Humanas da Universidade Federal de Santa Catarina, intitulada "A paisagem de Itapema: estudo da Geodiversidade para a Educação ambiental e o Geoturismo". No trabalho o autor teve como objetivo reconhecer a Geodiversidade de Itapema - Santa Catarina, a partir do estudo da paisagem, visando à implantação das práticas de educação ambiental e geoturismo na futura unidade de conservação do município (Covello, 2011).

Os trabalhos T5, T7 e T10, também acumulam enquadramentos com as categorias "Valoração/geoconservação" e "Educação ambiental”. Tratam-se de dissertações de mestrado. O T5, foi apresentado ao Programa de Pós-Graduação em Geografia da Universidade Estadual de Ponta Grossa, intitulado "Trilhas interpretativas como instrumento de Geoturismo e Geoconservação: caso da trilha do Salto São Jorge, Campos Gerais do Paraná”. No trabalho, o autor teve como objetivo destacar a importância das trilhas como instrumento de geoturismo e geoconservação, quando equipadas com os meios interpretativos adequados (Folmann, 2010).

O T7, apresentado ao Programa de Pós-Graduação em Sustentabilidade na Gestão Ambiental da Universidade Federal de São Carlos, intitulado "Inventário e quantificação dos Geossítios Cársticos do Parque Estadual Intervales (SP)” teve como seu objetivo, analisar 16 geossítios cársticos do Parque Estadual Intervales (PEI), possibilitando sua quantificação e valoração (Camargo, 2018).

Em T10, que foi apresentado ao Programa de Pós-Graduação em Geografia, da Universidade Federal de Londrina, na Linha de Pesquisa Dinâmica, Espaço-ambiental, intitulado "Geoturismo no norte paranaense: uma proposta de georoteiro para o município de Santo Antônio da Platina”, objetiva a discussão do trinômio, Geodiversidade, geoconservação e geoturismo, evidenciando a correlação entre esses conceitos, e buscando a elaboração de um roteiro geoturístico em seu município (Florentino Junior, 2018).

Os trabalhos T12, T13 e T14 correspondem somente a categoria "Geoturismo", e correspondem a dissertações de mestrado. O T12, apresentado ao Programa de Pós-Graduação em Patrimônio Cultural, Área de Concentração em Arquitetura e Patrimônio Material, na Linha de Pesquisa Arqueologia e Paleontologia, da Universidade Federal de Santa Maria, intitulado “Geoturismo: uma ferramenta auxiliar na interpretação e preservação do patrimônio geopaleontológico da região central do Rio Grande do Sul", teve por finalidade conhecer e entender a paleontologia da referida região e verificar a possibilidade deste patrimônio ser interpretado e preservado pelo viés do Geoturism. (Lorenci, 2013).

O T13, apresentado ao Programa de Pós-Graduação em Desenvolvimento e Meio Ambiente da Universidade Federal do Ceará, intitulado "Potencialidades para o desenvolvimento do geoturismo no município de Itapipoca (CE)" teve como principal objetivo analisar as potencialidades para o desenvolvimento do geoturismo no município de Itapipoca - CE (Machado, 2019).

O T14, apresentado ao Programa de Pós-Graduação em Turismo da Universidade Federal do Rio Grande do Norte, intitulado "Práticas turísticas em geossítios: uma avaliação ambiental no projeto Geoparque Seridó-RN" visava analisar a situação ambiental nos geossítios do Projeto Geoparque Seridó, em busca de uma prática turística de forma sustentável 
(Medeiros, 2015).

O trabalho T15 é o único nessa categoria que acumula enquadramentos com mais três outras: "Valoração/geoconservação", "Reconhecimento e Inventário" e "Educação ambiental". Trata-se da única tese de doutorado da presente pesquisa, apresentado ao Programa de Pós-Graduação em Geociências do Centro de Tecnologia e Geociências da Universidade Federal de Pernambuco, intitulada "Patrimônio geológico e estratégias de geoconservação: popularização das geociências e desenvolvimento territorial sustentável para o litoral sul de Pernambuco (Brasil)" no qual o objetivo do trabalho foi realizar a identificação dos principais elementos da Geodiversidade, seguido da inventariação do patrimônio geológico, com base em seus valores e especificidades (Guimarães, 2016).

\subsubsection{Reconhecimento e inventário}

Os trabalhos T1, T2, T6 e T15 foram enquadrados na categoria "Reconhecimento e inventário" pois em algum momento os mesmos citam palavras ou termos com esses sentidos. Os textos T1 e T15, ambas foram comentadas na categoria anterior (Geoturismo), pois acumulam enquadramento com essa e outras categorias.

O trabalho T2, trata-se de uma dissertação de mestrado apresentada ao Instituto de Geociências da Universidade Federal de São Paulo, intitulada "Avaliação da Geodiversidade em São Sebastião - SP, como patrimônio geológico" cujo principal objetivo do autor foi contribuir com o conhecimento, a conservação e a divulgação de parte do patrimônio geológico nacional através do inventário científico de interesse geológico do município de São Sebastião (Reverte, 2014)

O trabalho T6, acumula enquadramento com a categoria "Valoração/geoconservação", trata-se também de uma dissertação apresentada ao Instituto de Geociências e Ciências Exatas da Universidade Estadual Paulista, intitulada “Inventário, quantificação e valorização do geopatrimônio na Bacia do Rio Corumbataí (SP): subsídios ao Projeto Geoparque Corumbataí" na qual o principal objetivo foi contribuir para a adoção de estratégias de geoconservação, em apoio aos projetos de desenvolvimento sustentável na área de estudo (Kolya, 2019).

\subsubsection{Valoração/geoconservação}

Os trabalhos T4, T5, T6, T7, T9, T10, T11 e T15 foram enquadrados na categoria "Valoração/geoconservação" pois citam algumas palavras ou termos com esses sentidos. Os textos T5, T7, T10 e T15 foram comentados na categoria anterior, Geoturismo, T6 e T15 na categoria Reconhecimento e inventário, e estes acumulam enquadramento com essa e outras categorias.

O trabalho T4, que acumulam enquadramento com a categoria "Educação Ambiental", trata-se também de uma dissertação apresentada ao Programa de Pós-Graduação em Geociências da Universidade Federal do Rio Grande do Sul, intitulada "Geoconservação em grandes cidades e proposição dos itinerários geológicos de Porto Alegre: contribuições metodológicas para valoração integrada de unidades geológicas”. O objetivo do presente trabalho é apresentar contribuições metodológicas para geoconservação em ambientes urbanos, especificamente pela valoração integrada de unidades geológicas com base na análise de seis mapas éticos do município de Porto Alegre e pela proposição de uma matriz de valoração ponderada que relaciona 12 indicadores geopaisagísticos com as 26 unidades geológicas locais (Fontana, 2015).

Os trabalhos T9 e T11 são os únicos que nessa categoria não se enquadram cumulativamente com nenhuma outra. Ambos são dissertações de mestrado, em que o primeiro foi apresentado ao Programa de Pós-Graduação em Geociências do Instituto de Geociências da Universidade de São Paulo, intitulado "Estratégias de geoconservação no Parque Estadual da Serra do Mar - Núcleos Picinguaba e Caraguatatuba (SP)" cujo o objetivo foi definir estratégias de geoconservação pautadas em metodologias participativas nos núcleos Picinguaba e Caraguatatuba do Parque Estadual da Serra do Mar, a noroeste do estado de São Paulo. . (Mazzucato, 2017); e o segundo foi apresentado ao Programa de Pós-Graduação em Geografia da Universidade 
Federal de Santa Catarina, intitulado "O patrimônio geológico no desenvolvimento territorial em Tibagi, Paraná”, sob o objetivo de analisar a incorporação e implicações do patrimônio geológico no desenvolvimento de um território específico, o Município de Tibagi, localizado na região dos Campos Gerais do Paraná (Mochiutti, 2013).

\subsubsection{Educação Ambiental}

Os trabalhos T1, T4, T5, T7, T10 e T15 foram enquadrados na categoria "Educação Ambiental", pois todos os textos também foram citados aqui por se enquadrarem em alguma outra categoria, como T1, T5, T7, T10 e T15 na categoria Geoturismo; T1 e T15 na categoria Reconhecimento e inventário; T4, T5, T7, T10 e T15 na categoria Valoração/Geoconservação.

\subsubsection{As outras categorias}

Os demais trabalhos, T3, T8, T16 e T17, todas dissertações de mestrado, foram enquadrados cada um em uma única categoria sem reenquadramento cumulativo, a saber: Capital Cultural, Solos, Topografia e Caracterização Paisagística.

O trabalho T3, enquadrado na categoria Capital Cultural, apresentado ao programa de Pós-Graduação em Educação do Departamento de Educação do Centro de Teologia e Ciências Humanas da PUC-Rio, intitulado "Professores no Museu da Geodiversidade: o capital cultural nas percepções e expectativas da relação museu x escola”, que tem como objetivo mapear o volume de capital cultural dos professores que frequentaram o Museu da Geodiversidade (UFRJ) em visitas escolares entre os meses de maio e setembro de 2012, bem como suas percepções e expectativas acerca das instituições culturais, tais como o museu (Aracri, 2013).

O trabalho T8, enquadrado na categoria Solos, apresentado ao Programa de Pós-Graduação em Solos e Nutrição de Plantas da Universidade Federal de Viçosa, intitulado "Solos e alternância educativas: pesquisa-ação na formação de educadores", que teve como objetivo proporcionar aos estudantes que vieram do meio rural a possibilidade de permanecer e estudar em seu contexto sociogeográfico, de compreender melhor a sua realidade e de adquirir uma formação profissional voltada para as atividades agrícolas (Lobo, 2009).

O trabalho T16, enquadrado na categoria Topografia, apresentada ao Programa de Pós-Graduação em Ciência e Tecnologia Ambiental, área de concentração "Qualidade Ambiental”, da Universidade Federal do Triângulo Mineiro, intitulada "Compartimentos topomorfológicos e morfodinâmica da Bacia do Córrego das Lajes - Uberaba - Triângulo Mineiro" que tinha por objetivo utilizar as características físicas para identificar os compartimentos topomorfológicos da bacia do Córrego das Lajes, bem como verificar a ocorrência de basculamento na mesma (Martins Neto, 2018).

O trabalho T17, enquadrado na categoria Caracterização Paisagística, apresentado ao Programa de Mestrado de Pósgraduação em Geografia da Universidade Estadual do Oeste do Paraná, intitulado "Caracterização paisagística dos parques nacionais do Iguaçu e Iguazú", com objetivo de realizar a caracterização da Geodiversidade e da biodiversidade local bem como o processo de ocupação histórico, geográfico que compreende as relações estabelecidas no plano natureza, sociedade, para entender como a paisagem destes parques nacionais se estruturou ao longo do tempo e do espaço (Colpani, 2017).

\section{Discussão}

Apesar dos 17 trabalhos apresentados estarem vinculados a 15 instituições distribuídas no território brasileiro e levando em consideração a Geodiversidade nacional, não foi identificado nos trabalhos analisados, programas ou linhas de pesquisa de pós-graduação dedicadas a temática da "Geodiversidade aplicada ao ensino".

Apenas um entre todos os outros programas de pós-graduação foi efetivamente da área da Educação e o seu trabalho (T3), tratou de uma pesquisa sobre o mapeamento do volume de capital cultural dos professores que frequentaram o Museu da 
Geodiversidade (UFRJ) por meio de entrevistas semiestruturadas.

Vale ressaltar que, dos trabalhos aqui analisados, somente o T8 se destacou em relação aos outros trabalhos, pois o mesmo trata da construção, desenvolvimento e avaliação do curso denominado "Solos e Percepção Ambiental em Alternância”, oferecido pelo Programa de Educação em Solos e Meio Ambiente, abordando a perspectiva da pesquisa-ação com metodologias ativas, no que diz respeito na ação concreta da busca por soluções aos desafios existentes para o tema solos e alternância, cujo público alvo foram alunos, monitores e agricultores associados de uma Escola Família Agrícola.

\section{Considerações Finais}

A partir da análise das dissertações e teses de cursos de pós-graduação do Brasil entre os anos 2009 a 2019, pode-se concluir que a temática aqui abordada está presente em alguns trabalhos, mas de maneira ainda tímida e incipiente, especialmente quando se trata do ensino da Geodiversidade.

Acredita-se que isso se deve ao fato de que no país tem-se dado mais atenção ao conceito de Biodiversidade do que Geodiversidade, podendo ser por falta de conhecimentos, bem como por políticas educacionais "desatualizadas", pois nos documentos oficiais que direcionam a educação do país não há sequer uma citação da palavra Geodiversidade, apesar das inúmeras menções sobre assuntos relacionados ao meio ambiente.

Espera-se que, futuramente, com o aumento da popularidade do Geoturismo no país, aliada à Educação Ambiental, faça com que as autoridades competentes consigam perceber a carência de assuntos relacionados às Geociências na legislação, bem como em documentos normativos do ensino escolar brasileiro.

\section{Agradecimentos}

Os autores agradecem ao Núcleo de Pesquisa em Ciências Naturais do Semiárido do Piauí (Nupecinas). O presente trabalho foi realizado com apoio da Coordenação de Aperfeiçoamento de Pessoal de Nível Superior - Brasil (CAPES) - Código de Financiamento 001.

\section{Referências}

Alencar, R., Nascimento, R. S., \& Guimarães, G. B. (2012). Geociências no ensino fundamental: Ciências ou Geografia? da história da terra à paisagem local através da Geodiversidade da ilha de Santa Catarina [Resumo]. In III Simpósio Nacional de Ensino de Ciências e tecnologia (pp. 01-12). Ponta Grossa, Brasil.

Aracri, E. M. R. P. (2013). Professores no Museu da Geodiversidade: o capital cultural nas percepções e expectativas museu x escola. Dissertação de mestrado, Departamento de Educação do Centro de Tecnologia Ciências Humanas, PUC-Rio. Rio de Janeiro, Brasil.

Azevedo, Ú. R. (2007). Patrimônio geológico e geoconservação no Quadrilátero Ferrífero, Minas Gerais: potencial para a criação de um geoparque da UNESCO. Tese de doutorado, Programa de Pós-Graduação em Geologia, Universidade Federal de Minas Gerais, Belo Horizonte. Brasil.

Bardin, L. (1977). Análise de conteúdo (229pp). Lisboa: Edições 70.

Brilha, J. (2005). Geodiversidade. In Brilha, J. Patrimônio Geológico e Geoconservação - A conservação da natureza na sua vertente geológica (190pp). Braga: Palimage Editores.

Camargo, T. C. R. (2018). Inventário e quantificação dos geossítios cársticos do Parque Estadual de Intervales (SP). Dissertação de mestrado, Pós-Graduação em Sustentabilidade na Gestão Ambiental, Universidade Federal de São Carlos, Sorocaba. Brasil.

Carcavilla, L.; Durán, J. J.; Lopez-Martinez J. (2008). Geodiversidad: concepto y relación com el patrimonio geológico. In VII Congreso Geológico de España (pp. 1299-1303). Las Palmas de Gran Canaria, Espanha.

Colpani, J. (2017). Caracterização paisagística dos parques nacionais do Iguaçu e Iguazú. Dissertação de mestrado, Programa de Pós-Graduação em Geografia, Universidade Estadual do Oeste do Paraná, Marechal Cândido Rondon. Brasil.

Covello, C. (2012). A paisagem de Itapema: estudo da Geodiversidade para a educação ambiental e o geoturismo. Dissertação de mestrado, Programa de Pósgraduação em Geografia, Universidade Federal de Santa Catarina, Florianópolis, Brasil.

Florentino-Junior. E. (2018). Geoturismo no norte paranaense: uma proposta de georoteiro para o município de Santo Antônio da Platina. Dissertação de mestrado, Programa de Pós-graduação em Geografia, Universidade Estadual de Londrina,

Londrina, Brasil. 
Folmann, A. C. (2010). Trilhas interpretativas como instrumentos de geoturismo e geoconservação: caso da trilha do Salto de São Jorge, nos Campos Gerais do Paraná. Dissertação de mestrado, Programa de Pós-graduação em Geografia, Universidade Estatual de Ponta Grossa, Ponta Grossa, Brasil.

Fontana, R. C. (2015). Geoconservação em grandes cidades e proposição dos itinerários geológicos de Porto Alegre: contribuições metodológicas para valorização integrada de unidades geológicas. Dissertação de mestrado, Programa de Pós-graduação em Geociências, Universidade Federal do Rio Grande do Sul, Porto Alegre, Brasil.

Gray, M. (2004). Geodiversity and Valuing and Conserving Abiotic Nature (508pp). New York: John Wiley and Sons.

Guimarães. T. O. (2016). Patrimônio geológico e estratégias de geoconservação: popularização das geociências e desenvolvimento territorial sustentável para o litoral Sul de Pernambuco (Brasil). Tese de doutorado, Programa de Pós-graduação em Geociências, Universidade Federal do Pernambuco, Recife, Brasil.

Kiernan, K. (2001). The Geomorphology and Geoconservation Significance of Lake Pedder. In Sharples, C. (Ed.) Lake Pedder: Values and Restoration (pp. 13-50). University of Tasmania.

Kolya, A. A. (2019). Inventário, quantificação e valorização do geopatrimônio na Bacia do Rio Corumbataí (SP): subsídios ao projeto Geoparque Corumbataí. Dissertação de mestrado, Pós-Graduação em Geociências e Meio Ambiente, Universidade Estadual Paulista, Rio Claro, Brasil.

Lobo, L. M. (2009). Solos e alternâncias educativas: pesquisa-ação na formação de educadores. Dissertação de mestrado, Pós-Graduação em Solos e Nutrição de Plantas, Universidade Federal de Viçosa, Viçosa, Brasil.

Lorenci, C. T. B. (2013). Geoturismo: uma ferramenta auxiliar na interpretação e preservação do patrimônio geopaleontológico da região central do Rio Grande do Sul. Santa Maria. Dissertação de mestrado, Pós-Graduação Profissionalizante em Patrimônio Cultural, Universidade Federal de Santa Maria, Santa Maria, Brasil

Machado, R. M. (2019). Potencialidades para o desenvolvimento do geoturismo no Município de Itapipoca (CE).

Dissertação de mestrado, Programa de Pós-graduação em desenvolvimento e meio ambiente, Universidade Federal do Ceará, Fortaleza, Brasil.

Martins-Neto, F. F. (2018). Compartimentos topomorfológicos e morfodinâmicos da Bacia do Córrego das Lajes - Uberaba - Triângulo Mineiro. Dissertação de mestrado, Programa de Pós-Graduação em Ciência e Tecnologia Ambienta, Universidade Federal do Triângulo Mineiro, Uberaba, Brasil.

Mazzucato, E. (2017). Estratégias de geoconservação no Parque Estadual da Serra do Mar - Núcleo Picinguaba e Caraguatatuba (SP). Dissertação de mestrado, Programa de Pós-Graduação em Geociências, Universidade de São Paulo, São Paulo, Brasil.

Medeiros, J. L. (2015). Práticas turísticas em geossítios: uma avaliação ambiental no projeto Geoparque Seridó-RN. Dissertação de mestrado, Programa de Pós-Graduação em Turismo, Universidade Federal do Rio Grande do Norte, Natal, Brasil.

Mochiutti, N. F. (2013). O patrimônio geológico no desenvolvimento territorial em Tibagi, Paraná. Florianópolis. Dissertação de mestrado, Programa de PósGraduação em Geografia, Universidade Federal de Santa Catarina, Florianópolis, Brasil.

Reverte, F. C. (2014). Avaliação da Geodiversidade em São Sebastião - SP, como patrimônio geológico. Dissertação de mestrado, Programa de Pósgraduação em Geologia, Universidade de São Paulo, São Paulo, Brasil.

Serrano-Cañadas, S.; Ruyz-Flaño, P. (2007). Geodiversidad: Concepto, Evaluación y Aplicación Territorial. El caso de tiermes Caracena (Soria). Boletín de la A.G.E. 45(1).

Sharples, C. (2002). Concepts and Principles of Geoconservation. Tasmanian Parks \& Wildlife Service.

Souza, A. R.; Machado, D. M. C.; Ponciano, L. C. M. O.; Faria, A. C. G.; Vieira, A. C. M.; Pinto, F. M. (2007). Geoconservação: A preservação e valorização do patrimônio geológico. In Ed. Carvalho, I. S. Paleontologia: senários de vida. (pp. 652) Editora Interciência. Rio de Janeiro.

Xavier, L. S.; Meneses, L. F.; Cavalcante, M.; B. (2017). Ensinando Geodiversidade a partir de jogos didáticos. Revista de Ensino de Geografia, Uberlândia, 15(8), 157-182. 